\title{
Short- and long-term outcomes after postsurgical acute kidney injury requiring dialysis
}

This article was published in the following Dove Press journal:

Clinical Epidemiology

\author{
Yu-Feng Lin ${ }^{1,2}$ \\ Tao-Min Huang 2,3 \\ Shuei-Liong $\operatorname{Lin}^{2,4}$ \\ Vin-Cent $\mathrm{Wu}^{2}$ \\ Kwan-Dun $\mathrm{Wu}^{2}$ \\ 'Graduate Institute of Clinical \\ Medicine, National Taiwan University \\ College of Medicine, Taipei, Taiwan; \\ ${ }^{2}$ Division of Nephrology, Department \\ of Internal Medicine, National Taiwan \\ University Hospital, Taipei, Taiwan; \\ ${ }^{3}$ Graduate Institute of Epidemiology \\ and Preventive Medicine, National \\ Taiwan University College of Public \\ Health, Taipei, Taiwan; ${ }^{4}$ Graduate \\ Institute of Physiology, National \\ Taiwan University College of \\ Medicine, Taipei, Taiwan
}

Correspondence: Vin-Cent Wu Department of Internal Medicine, National Taiwan University Hospital, 7 Chung-Shan South Road, Taipei, 10002, Taiwan

Email q91421028@ntu.edu.tw
Objective: Prompt assessment of perioperative complications is critical for the comprehensive care of surgical patients. Acute kidney injury requiring dialysis (AKI-D) is associated with high mortality, yet little is known about how long-term outcomes of patients have evolved. The association of AKI-D with postsurgical outcomes has not been well studied.

Methods: We investigated patients from the National Health Insurance Research Database and validated by the multicenter Clinical Trial Consortium for Renal Diseases cohort. All patients with AKI-D 18 years or older undergoing four major surgeries (cardiothoracic, esophagus, intestine, and liver) were retrospectively investigated $(\mathrm{N}=106,573)$. Patient demographics, surgery type, comorbidities before admission, and postsurgical outcomes, including the in-hospital, 30-day, and long-term mortality together with dialysis dependence were collected.

Results: AKI-D is the top risk factor for 30-day and long-term mortality after major surgery. Of 1,664 individuals with AKI-D and 6,656 matched controls, AKI-D during the hospital stay was associated with in-hospital (adjusted hazard ratio [aHR] $=3.04,95 \%$ CI 2.79-3.31), 30-day (aHR=3.65, 95\% CI 3.37-3.94), and long-term (aHR=3.22, 95\% CI 3.01-3.44) mortality. Patients undergoing cardiothoracic surgery (CTS) showed less in-hospital (aHR=0.85, 95\% CI $0.75-0.97), 30$-day $(\mathrm{aHR}=0.79,95 \%$ CI $0.70-0.89)$, and long-term $(\mathrm{aHR}=0.80,95 \% \mathrm{CI}$ 0.72-0.90) mortality compared with non-CTS patients with AKI-D. CTS patients had a high risk of 30-day dialysis dependence (subhazard ratio $[\mathrm{sHR}]=1.67,95 \% \mathrm{CI} 1.18-2.38$ ), but the risk of long-term dialysis dependence was similar ( $\mathrm{sHR}=1.38,95 \%$ CI 0.96-2.00) after AKI-D by taking mortality as a competing risk. Non-CTS patients had more comorbidities of sepsis, azotemia, hypoalbuminemia, and metabolic acidosis compared with CTS patients.

Conclusion: AKI exhibits paramount effects on postsurgical outcomes that extend well beyond discharge from the hospital. The goal of the perioperative assessment should include the reassurance of enhancing renal function recovery among different surgeries, and optimized follow-up is warranted in attenuating the complications after postsurgical AKI has occurred.

Keywords: major surgery, acute kidney injury, postsurgical complication, dialysis dependence, mortality

\section{Introduction}

Acute kidney injury (AKI) is a severe perioperative complication. ${ }^{1,2}$ Although the incidence of postsurgical AKI depends immensely on the type of the surgery, up to $40 \%$ of hospital-acquired AKI is related to the perioperative setting. ${ }^{3,4}$ It is associated with prolonged hospital stay, increased costs, and both short- and long-term mortality. ${ }^{5-8}$ Among the patients with the most severe forms of $\mathrm{AKI}$, those requiring dialysis (AKI-D) experience higher mortality ${ }^{9}$ and incur greater resource use. ${ }^{10,11}$ Additionally, 
postsurgical AKI is associated with long-term adverse events including mortality and the development of chronic kidney disease. ${ }^{12-14}$ AKI can be a reflection of the general insult of critical illness while also being an independent contributor to that illness; postsurgical AKI is of particular interest as a significant and measurable event of perioperative harm and a plausible target for intervention. Severe AKI after a major surgery is associated with a high risk of mortality and increased costs of care; this adverse impact might extend beyond the discharge of such surgical patients from the hospital. ${ }^{15}$

Recently, the American College of Surgeons National Surgical Quality Improvement Program (ACS-NSQIP) ${ }^{16}$ was established based on a "surgery-target" approach for improving risk-adjusted surgical outcomes and allowing highly detailed evaluations of performance and zero in on preventable complications. ${ }^{17,18}$ Because a growing movement aims to tie reimbursements to outcomes, the performance related to the influence of postsurgical AKI among different surgeries to in-hospital, 30-day, and long-term mortality as well as dialysis dependence is important for enhancing quality initiatives. To our knowledge, the association between surgical type and 30-day or long-term dialysis dependence together with the clinical significance of AKI episodes with respect to clinically relevant outcomes has not been comprehensively studied in a surgical patient population.

This study aimed to evaluate the influence of AKI-D on postsurgical in-hospital, 30-day, and long-term outcomes, including mortality and dialysis dependence, among patients who were undergoing different major surgeries. We investigated the association between AKI-D and outcomes while adjusting for surgery procedures, patient demographics, and comorbidities, using a large nationwide database and validated by prospectively enrolled nationwide cohort.

\section{Methods}

\section{Data source}

The National Health Insurance Research Database (NHIRD) of the National Health Insurance (NHI) program is mandatory and universal, offering comprehensive medical care coverage to more than $99 \%$ of the country's population of 23 million people. The NHI system contains all original claims data, including demographic information and detailed orders, for all clinic visits and hospitalizations. Disease diagnoses for all individuals are classified according to the ICD, Ninth Revision, Clinical Modification (ICD-9-CM). Taiwan's NHI records are regularly inspected, and physicians who violate the standards of clinical practice are subject to financial penalties. ${ }^{19}$ The accuracy of diagnoses registered in the NHIRD has been validated. ${ }^{20,21}$

For this study, patients (aged $\geq 18$ years) who were admitted to the hospital from January 2001 to December 2007 for major surgery and who developed AKI-D during their index admission were identified in the NHI databases. Surgical procedures were considered major if the length of the intensive care unit (ICU) stay for patients in a given diagnosis-related group exceeded 2 days. ${ }^{22}$ Major surgery procedures were further classified into cardiothoracic, esophageal, intestinal, and hepatic as the primary diagnosis codes. ${ }^{4}$ We further divided all major surgeries into cardiothoracic surgery (CTS) and non-CTS. Because the identification numbers of all individuals in the NHRID were encrypted to protect the privacy of the individuals and informed consent was waived due to the secondary nature of the patient data, this study underwent limited ethical review by the Institutional Review Board of National Taiwan University Hospital (approval reference no. 201212021RINC).

\section{Validation cohort}

The Taiwan Consortium for Acute Kidney Injury and Renal Diseases (CAKS) from the National Research Program for Biopharmaceuticals has launched a nationwide epidemiology and prognostic program for tracking AKI-D, which prospectively enrolls critically ill patients with AKI-D in ICUs. CAKS includes 30-member hospitals that are widely distributed throughout the four geographical regions (north, middle, south, and east) of Taiwan. The CAKS member hospitals have a 1:1 ratio of medical centers to regional hospitals in each region and in the four seasonally sampled months (October 2014 and January, April, and July 2015). ${ }^{23}$ Patients undergoing dialysis after major surgery were selected to be in the validation cohort. This cohort included 79 CTS patients and 154 non-CTS patients. We used this cohort to validate different clinical manifestations and laboratory data between CTS patients and non-CTS patients during AKI-D. Detailed laboratory data and scoring systems were compared in each group of patients at ICU admission and the initiation of renal replacement therapy (RRT).

\section{Data collection and outcome measures}

Patient demographics, surgery type, Charlson comorbidity index, comorbidities before admission, and postsurgical outcomes including the in-hospital, 30-day, and long-term mortality together with dialysis dependence were reviewed from the database. The primary outcomes were in-hospital, 
30-day, and long-term mortality after hospital discharge. The secondary outcomes were 30-day and long-term dialysis dependence defined as the requirement for dialysis for at least 3 months after hospital discharge, with mortality treated as a competing risk. Composite outcomes combined the outcomes of mortality and dialysis dependence. Each patient was monitored from the date of discharge to 30 days after discharge for 30-day mortality and was censored at either death or the end of the study (December 31, 2010) for long-term outcome, whichever occurred first.

\section{Statistical analyses}

We followed the STROBE (Strengthening the Reporting of Observational Studies in Epidemiology) recommendations for this study. After discharge, the status of dialysis dependence was integrated as a time-varying risk.

We performed a propensity score matching (PSM) to reduce the selection bias due to the baseline differences between the patients of AKI with dialysis and those of AKI without dialysis. First, we conducted multivariate logistic regression analysis to identify the predictors for the prescription of dialysis. Using logistic regression model, factors during the index hospitalization as predictors of AKI-D including age, sex, surgical type, hospital stay length, Charlson comorbidity index score, comorbidities, and postsurgical complications were identified as parameters for further PSM (Table S1 and Figures S2). Then, we used the "Match()" function in the "Matching" library of the R statistical software to do PSM based on the default value of "caliper" (ie, caliper $=0.25$ ) and the Mahalanobis distance without replacement. After the matching, the standardized mean difference of all variables was $<0.1$ (Figure S2).

Patients who did not undergo acute dialysis during their index hospitalization but had a major surgery were matched to the study group at a 1:4 ratio after matching for age, sex, Charlson comorbidity index, and propensity score. We first modeled 30-day and long-term mortality using the occurrence of AKI-D after the surgery as the primary covariate of interest and adjusted for surgery type and preoperative covariates, including age, sex, Charlson comorbidity index, and individual comorbidities. However, cardiac surgery had the highest postoperative AKI risk. ${ }^{24}$ In addition, acute renal failure after cardiac surgery is known to be associated with significant short-term morbidity and mortality. ${ }^{25}$ In light of this, we constructed a second model stratifying AKI-D patients into CTS and non-CTS while adjusting the same preoperative covariates as the first model. In further parametric modeling with regard to factors associated with outcome, we adopted three modeling methods: simple Cox regression, multivariable Cox regression, and the competing risk regression. A Cox proportional regression model was used to calculate the adjusted hazard ratios (aHRs) and 95\% CIs for the outcomes including in-hospital, 30-day, and long-term mortality and dialysis dependence. Because of high mortality and dialysis dependence rates in surgical patients with AKI-D, competing risk regression applying Fine and Gray modeling for consideration of the subdistribution hazard was performed to model dialysis dependence while treating mortality as a competing risk. ${ }^{26}$ Furthermore, the validation cohort was analyzed by PSM at a 1:1 ratio after matching for comorbidities and baseline demographics including age, sex, and severity scores. A bivariate analysis was performed using the $t$-test for normally distributed continuous variables and Wilcoxon rank-sum test for nonnormally distributed continuous variables, and a chi-squared analysis was used for categorical variable comparisons and outcome events. Missing or invalid data on age, sex, and related variables were noted in $1.2 \%$ of the patients, and these patients were excluded from the analyses. We used R software, version 2.8.1 (Free Software Foundation, Inc., Boston, MA, USA); competing risk analysis was performed with Stata MP, version 12 (StataCorp, College Station, TX, USA). A two-sided $P$-value $<0.05$ was considered statistically significant.

\section{Results \\ Data source and participants}

From 2001 to 2007, 112,745 patients underwent major surgeries and 1,861 surgical patients with AKI-D during their index hospitalization were enrolled in the study (Figure 1). The incidence of AKI-D after major surgeries increased during this period, and the aHR of 30-day and long-term mortality also increased simultaneously (Figure S1 and Table S2). Compared with all the risk factors, AKI-D was the top risk factor for 30-day and long-term mortality (Table S3). With PSM at a 1:4 ratio, 8,320 surgical patients, 1,664 with AKI-D and 6,656 without dialysis were further analyzed.

\section{Demographic characteristics, comorbidities, postsurgical complications, and outcomes}

Baseline characteristics stratified by the AKI-D, surgery type, and comorbidities before and after the PSM are presented in Table 1. Of all patients undergoing major surgeries, the AKI-D group had the most underlying diseases and 


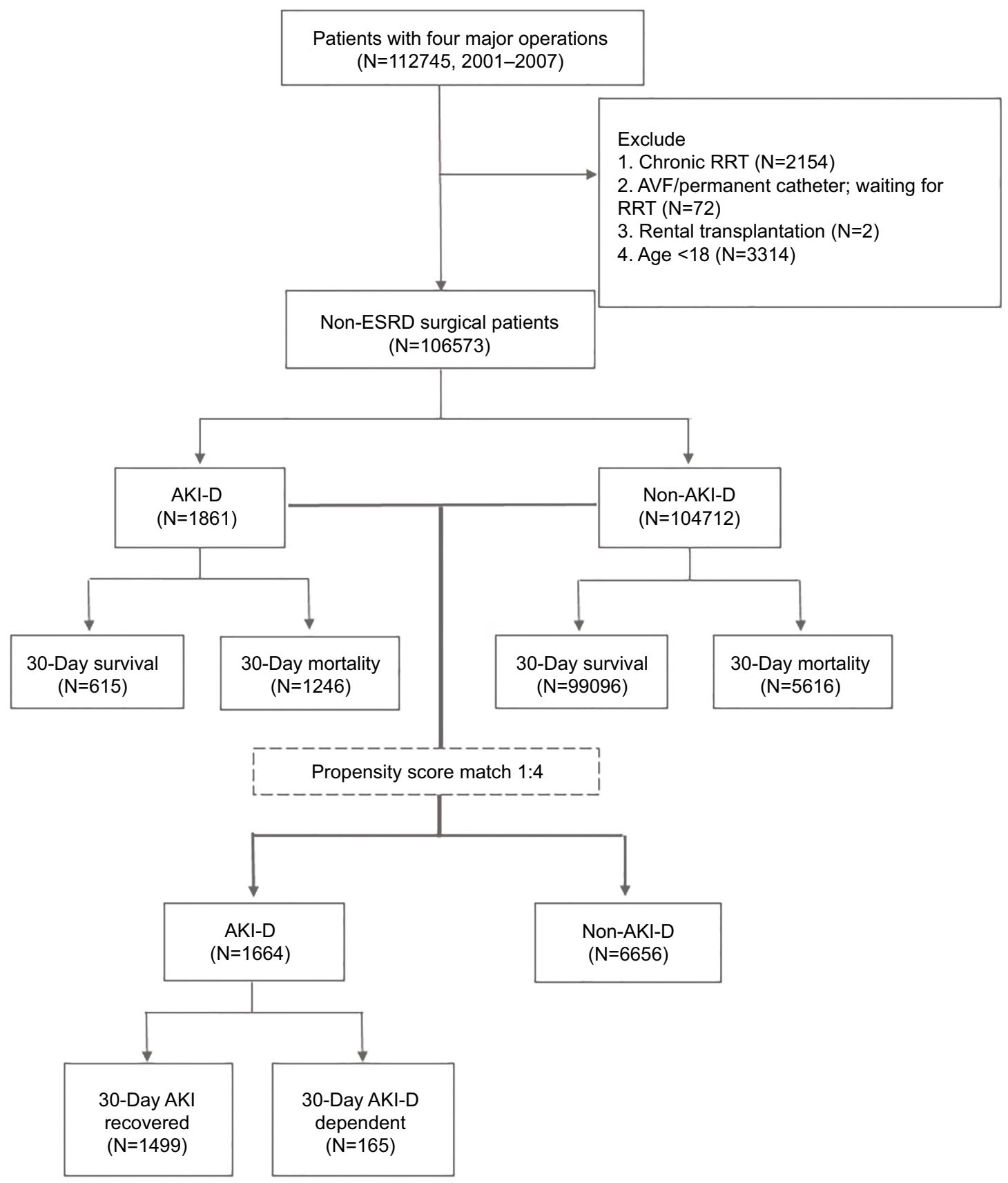

Figure I Study flow diagram.

Abbreviations: AKI, acute kidney injury; AKI-D, acute kidney injury requiring dialysis; AVF, arteriovenous fistula; ESRD, end-stage renal disease; RRT, renal replacement therapy.

postsurgical comorbidities compared with the non-AKI-D group (Table 1). Besides, the surgical patients with AKI-D had higher in-hospital mortality compared with the nonAKI-D surgical patients (66.95\% vs 5.37\%). After the PSM (Table S1 and Figures S2), the surgical patients with AKI-D still had higher in-hospital mortality compared with the nonAKI-D surgical patients (68.87\% vs $22 \cdot 64 \%)$.

\section{All-cause mortality after postsurgical AKI-D}

After PSM, 8,320 surgical patients were analyzed using time-dependent Cox proportional hazard modeling, with dialysis dependence after hospital discharge as the timevarying risk. Similar results were obtained before and after PSM. After matching with the adjustment of baseline 
Table I Basic demographic characteristics, comorbidities, complications, and outcomes in surgical patients stratified by AKI-D during the hospital stay

\begin{tabular}{|c|c|c|c|c|c|c|c|}
\hline \multirow[t]{2}{*}{ Characteristic } & \multirow{2}{*}{$\begin{array}{l}\text { All } \\
n=106,573\end{array}$} & \multirow{2}{*}{$\begin{array}{l}\text { AKI-D } \\
n=I, 86 I\end{array}$} & \multirow{2}{*}{$\begin{array}{l}\text { Non-AKI-D } \\
n=104,7 \mid 2\end{array}$} & \multirow[t]{2}{*}{ SMD } & \multicolumn{3}{|c|}{ Propensity score-matched } \\
\hline & & & & & $\begin{array}{l}\text { AKI-D } \\
\mathrm{n}=1,664\end{array}$ & $\begin{array}{l}\text { Non-AKI-D } \\
n=6,656\end{array}$ & SMD \\
\hline Male sex & $60,847(57.09 \%)$ & $\mathrm{I}, 200(64.48 \%)$ & $59,647(56.96 \%)$ & 0.154 & $\mathrm{I}, 075(64.60 \%)$ & $4,440(66.71 \%)$ & -0.044 \\
\hline Age at admission & $58.72 \pm 16.88$ & $65.91 \pm 15.33$ & $58.59 \pm 16.88$ & -0.454 & $65.67 \pm 15.62$ & $66.56 \pm|4.7|$ & 0.058 \\
\hline \multicolumn{8}{|l|}{ Surgical type } \\
\hline Cardiothoracic & 20,335 (19.08\%) & $859(46.15 \%)$ & $19,476(18.60 \%)$ & -0.276 & $723(43.45 \%)$ & $3,033(45.57 \%)$ & 0.021 \\
\hline Esophagus & $18,268(17.14 \%)$ & 347 (18.65\%) & $|7,92|(|7| 1 \%)$. & -0.026 & 331 (19.89\%) & $1,239(18.61 \%)$ & -0.021 \\
\hline Intestine & 27,657 (25.95\%) & 404 (21.71\%) & $27,253(26.03 \%)$ & 0.077 & $372(22.36 \%)$ & 1,329 (19.97\%) & -0.043 \\
\hline Liver & 40,3 I3 (37.83\%) & 251 (I3.49\%) & $40,062(38.26 \%)$ & 0.575 & $238(14.30 \%)$ & $\mathrm{I}, 055(\mathrm{I} 5.85 \%)$ & 0.042 \\
\hline \multicolumn{8}{|l|}{ Comorbidities (before admission) } \\
\hline Myocardial infarction & I,658 (I.56\%) & 119 (6.39\%) & I,539 (I.47\%) & 0.255 & 95 (5.7I\%) & 394 (5.92\%) & -0.009 \\
\hline Congestive heart failure & $3,678(3.45 \%)$ & $283(15.21 \%)$ & $3,395(3.24 \%)$ & 0.423 & $226(13.58 \%)$ & 835 (I2.55\%) & 0.031 \\
\hline Peripheral vascular disease & $807(0.76 \%)$ & $35(1.88 \%)$ & $772(0.74 \%)$ & 0.101 & $29(1.74 \%)$ & 166 (2.49\%) & -0.052 \\
\hline Cerebrovascular disease & $4,984(4.68 \%)$ & |4| (7.58\%) & $4,843(4.63 \%)$ & 0.124 & 119 (7.15\%) & $506(7.60 \%)$ & -0.017 \\
\hline Dementia & I,074 (I.0I\%) & $34(1.83 \%)$ & I,040 (0.99\%) & 0.071 & 32 (1.92\%) & I55 (2.33\%) & -0.028 \\
\hline COPD & $9,318(8.74 \%)$ & 244 (13.1I\%) & $9,074(8.67 \%)$ & 0.143 & $219(13.16 \%)$ & 929 (13.96\%) & -0.023 \\
\hline Rheumatologic disease & $612(8.74 \%)$ & 27 (I3.II\%) & $585(8.67 \%)$ & 0.090 & $21(1.26 \%)$ & $96(1.44 \%)$ & -0.016 \\
\hline Peptic ulcer disease & 18,919 (17.75\%) & $326(17.52 \%)$ & I8,593 (17.76\%) & -0.006 & $289(17.37 \%)$ & $\mathrm{I}, \mathrm{I} 66(17.52 \%)$ & -0.004 \\
\hline Mild liver disease & 8,185 (7.68\%) & 175 (9.40\%) & 8,010 (7.65\%) & 0.063 & 153 (9.19\%) & $612(9.19 \%)$ & 0.000 \\
\hline Diabetes & 14,127 (I3.26\%) & $546(29.34 \%)$ & $|3,58|(\mid 2.97 \%)$ & 0.409 & $452(27.16 \%)$ & 1965 (29.52\%) & -0.052 \\
\hline Hemiplegia or paraplegia & $409(0.38 \%)$ & $8(0.43 \%)$ & $40 I(0.38 \%)$ & 0.007 & $6(0.36 \%)$ & $28(0.42 \%)$ & -0.010 \\
\hline Malignancy & $4,405(4.13 \%)$ & $80(4.30 \%)$ & $4,325(4.13 \%)$ & 0.008 & $76(4.57 \%)$ & $275(4.13 \%)$ & 0.021 \\
\hline Moderate or severe liver disease & $897(0.84 \%)$ & $54(2.90 \%)$ & $843(0.81 \%)$ & 0.156 & $49(2.94 \%)$ & $176(2.64 \%)$ & 0.018 \\
\hline AIDS & $15(0.01 \%)$ & $0(0.00 \%)$ & $15(0.01 \%)$ & -0.017 & $0(0.00 \%)$ & $3(0.05 \%)$ & -0.030 \\
\hline Charlson comorbidity index score & $0.89 \pm 1.39$ & $\mathrm{I} .74 \pm \mathrm{I} .8 \mathrm{I}$ & $0.88 \pm 1.37$ & -0.537 & $1.59 \pm 1.72$ & $1.69 \pm 1.87$ & 0.057 \\
\hline CKD & I, 197 (1.12\%) & 218 (11.71\%) & 979 (0.93\%) & 0.454 & $130(7.81 \%)$ & 447 (6.72\%) & 0.042 \\
\hline \multicolumn{8}{|l|}{ Comorbidities (during index } \\
\hline \multicolumn{8}{|l|}{ hospitalization) } \\
\hline \multicolumn{8}{|l|}{ Pulmonary complications } \\
\hline Reintubation & $157(0.14 \%)$ & $40(2.15 \%)$ & II 7 (0.1 I\%) & 0.194 & $29(1.74 \%)$ & $87(1.31 \%)$ & 0.036 \\
\hline $\begin{array}{l}\text { Acute respiratory distress } \\
\text { syndrome }\end{array}$ & $4,402(4.13 \%)$ & 738 (39.66\%) & $3,664(3.50 \%)$ & 0.979 & $603(36.24 \%)$ & $2,24 \mathrm{I}(33.67 \%)$ & 0.054 \\
\hline Pleural effusion & I,927 (I.8I\%) & 78 (4.19\%) & I,849 (I.77\%) & 0.143 & $7 \mathrm{I}(4.27 \%)$ & $298(4.48 \%)$ & -0.010 \\
\hline Chest tube insertion & $3,536(3.32 \%)$ & $315(16.93 \%)$ & 3,221 (3.08\%) & 0.474 & $253(15.20 \%)$ & $952(14.30 \%)$ & 0.025 \\
\hline \multicolumn{8}{|l|}{ Cardiac complications } \\
\hline Hemopericardium & $693(0.65 \%)$ & 37 (1.99\%) & $656(0.63 \%)$ & 0.120 & 33 (1.98\%) & 144 (2.16\%) & -0.013 \\
\hline Hypovolemic shock & $2,277(2.14 \%)$ & $268(14.40 \%)$ & $2,009(1.92 \%)$ & 0.468 & $223(13.40 \%)$ & 831 (12.48\%) & 0.027 \\
\hline Cardiac arrest & $5 \mathrm{I}(0.05 \%)$ & $9(0.48 \%)$ & 42 (0.04\%) & 0.087 & 7 (0.42\%) & 16 (0.24\%) & 0.031 \\
\hline Heart block & $274(0.26 \%)$ & II (0.59\%) & $263(0.25 \%)$ & 0.053 & $8(0.48 \%)$ & $31(0.47 \%)$ & 0.002 \\
\hline Atrial fibrillation & $2,984(2.80 \%)$ & $92(4.94 \%)$ & $2,892(2.76 \%)$ & 0.114 & $8 \mathrm{I}(4.87 \%)$ & $355(5.33 \%)$ & -0.021 \\
\hline ECMO & $5,050(4.74 \%)$ & 509 (27.35\%) & 4,54 I (4.34\%) & 0.664 & 414 (24.88\%) & I,692 (25.42\%) & -0.012 \\
\hline \multicolumn{8}{|l|}{ Infectious complications } \\
\hline Pneumonia & 4,377 (4.II\%) & 355 (19.08\%) & $4,022(3.84 \%)$ & 0.493 & $293(17.61 \%)$ & $\mathrm{I}, \mathrm{I} 50$ (17.28\%) & 0.009 \\
\hline Urinary tract infection & $2,504(2.35 \%)$ & 100 (5.37\%) & $2,404(2.30 \%)$ & 0.161 & $80(4.81 \%)$ & $386(5.80 \%)$ & -0.044 \\
\hline Severe sepsis & $2,533(2.38 \%)$ & $387(20.80 \%)$ & $2,146(2.05 \%)$ & 0.617 & $319(19.17 \%)$ & I,220 (I8.33\%) & 0.022 \\
\hline \multicolumn{8}{|l|}{ Others } \\
\hline Delirium & 180 (0.17\%) & $5(0.27 \%)$ & I75 (0.17\%) & 0.022 & $5(0.30 \%)$ & 22 (0.33\%) & -0.005 \\
\hline Stroke & I, 198 (1.12\%) & $48(2.58 \%)$ & $1,150(1.10 \%)$ & 0.110 & 39 (2.34\%) & $157(2.36 \%)$ & -0.001 \\
\hline Gl bleeding & $2,733(2.56 \%)$ & $142(7.63 \%)$ & $2,59 \mid$ (2.47\%) & 0.237 & $123(7.39 \%)$ & 515 (7.74\%) & -0.013 \\
\hline
\end{tabular}

Note: Data presented as $\mathrm{n}(\%)$ or mean \pm SD.

Abbreviations: AKI-D, acute kidney injury requiring dialysis; CKD, chronic kidney disease; COPD, chronic obstructive pulmonary disease; ECMO, extracorporeal membrane oxygenation; GI, gastrointestinal; SMD, standardized mean difference.

demographics, surgery type, Charlson comorbidity index, and comorbidities, postsurgical AKI-D augmented inhospital (aHR: 3.04, 95\% CI 2.79-3.31), 30-day (aHR:
3.65, 95\% CI 3.37-3.94), and long-term (aHR: $3.22,95 \%$ CI 3.01-3.44) mortality after a median follow-up of 294.5 days (Table 2). 
Table 2 Comparison of the in-hospital, 30-day, and long-term mortality in AKI-D vs non-AKI-D in surgical patients before and after propensity score matching ${ }^{\mathrm{a}}$

\begin{tabular}{|c|c|c|c|c|c|c|c|c|c|}
\hline & Mortality & Events & $\begin{array}{l}\text { Person- } \\
\text { years }\end{array}$ & $\begin{array}{l}\text { Incidence } \\
\text { (per I,000 } \\
\text { person-years) }\end{array}$ & Events & $\begin{array}{l}\text { Person- } \\
\text { years }\end{array}$ & $\begin{array}{l}\text { Incidence } \\
\text { (per I,000 } \\
\text { person-years) }\end{array}$ & $\begin{array}{l}\text { Crude hazard } \\
\text { ratio }(95 \% \mathrm{Cl})\end{array}$ & $\begin{array}{l}\text { Adjust hazard } \\
\text { ratio }^{\mathrm{b}}(95 \% \mathrm{Cl})\end{array}$ \\
\hline & & \multicolumn{3}{|l|}{ AKI-D } & \multicolumn{3}{|c|}{ Non-AKI-D } & & \\
\hline Before & In-hospital & $\mathrm{I}, 084$ & 90,201 & 12 & 4,066 & $\mathrm{I}, 707,808$ & 2.4 & $3.64(3.39-3.91)$ & $3.37(3.13-3.62)$ \\
\hline \multirow[t]{3}{*}{ matching } & 30-day & 1,246 & 109,626 & 11.4 & 5,616 & $4,698,184$ & 1.2 & $7.63(7.13-8.16)$ & $6.03(5.64-6.45)$ \\
\hline & Long-term & 1,439 & 503,341 & 2.9 & 24,197 & $104,893,286$ & 02 & $8.72(8.26-9.20)$ & $5.79(5.48-6.11)$ \\
\hline & & \multicolumn{3}{|l|}{ AKI-D } & \multicolumn{3}{|c|}{ Non-AKI-D } & & \\
\hline$\overline{\text { After }}$ & In-hospital & 1,006 & 74,417 & 13.5 & $I, 16 \mid$ & 256,992 & 4.5 & $3.00(2.76-3.27)$ & $3.04(2.79-3.31)$ \\
\hline \multirow[t]{2}{*}{ matching } & 30-day & 1,146 & 90,744 & 12.6 & $\mathrm{I}, 507$ & 415,220 & 3.6 & $3.58(3.31-3.87)$ & $3.65(3.37-3.94)$ \\
\hline & Long-term & 1,300 & 436,476 & 3 & 3,065 & $4,179,988$ & 0.7 & $3.08(2.88-3.29)$ & $3.22(3.0 \mathrm{I}-3.44)$ \\
\hline
\end{tabular}

Notes: a This model showed good validity, with the adjusted generalized $R^{2}$ and the concordance index being 0.4142 and 0.86 , respectively. ${ }^{b} \mathrm{Adjusted}$ by age, sex, surgery type, Charlson comorbidity index, and individual comorbidities.

Abbreviation: AKI-D, acute kidney injury requiring dialysis.

\section{Competing risk models of the outcomes}

The competing risk model for stratifying AKI-D patients into CTS and non-CTS patients was additionally adjusted for age, sex, Charlson comorbidity index, individual comorbidities, and propensity score. We found similar results before and after PSM. After the matching, patients who underwent CTS had lower in-hospital (aHR: 0.85, 95\% CI 0.75-0.97), 30-day (aHR: 0.79, 95\% CI 0.70-0.89), and long-term mortality (aHR: 0.80, 95\% CI 0.72-0.90); higher 30-day dialysis dependence (competing subhazard ratio [sHR]: 1.67, 95\% CI 1.18-2.38); and similar long-term dialysis dependence (sHR: $1.38,95 \%$ CI 0.96-2.00) compared with non-CTS patients after accounting for mortality as a competing risk (Table 3 and Figure 2). Furthermore, CTS patients had better 30-day (aHR: 0.85, 95\% CI 0.76-0.96) and long-term (aHR: $0.81,95 \%$ CI $0.73-0.91$ ) composite outcome than non-CTS patients (Table 3).

\section{Clinical manifestations of postsurgical AKI}

More detailed laboratory data of CTS and non-CTS patients were compared across all hospitals in the CAKS cohor ${ }^{23}$ for the validation of different clinical manifestations at postsurgical AKI during hospitalization. After PSM at a 1:1 ratio (Table S4), 144 patients ( 72 CTS patients, 72 non-CTS patients) were further analyzed. Non-CTS patients had more comorbidities of sepsis and azotemia during index hospitalization, together with more hypoalbuminemia and more metabolic acidosis compared with CTS patients (Table 4). Furthermore, non-CTS patients tend to receive intermittent hemodialysis, while CTS patients tend to receive continuous venovenous hemofiltration during acute kidney injury requiring dialysis (Table 4).

\section{Discussion}

Postsurgical AKI-D was associated with in-hospital, 30-day, and long-term mortality in patients undergoing major surgeries. In the analysis of surgery type, AKI-D patients who underwent CTS had lower in-hospital, 30-day, and long-term mortality compared with patients who had other types of surgeries. However, CTS patients with AKI-D had a higher risk of 30-day dialysis dependence but a similar risk of longterm dialysis dependence compared with non-CTS patients. This study showed that AKI-D in CTS and non-CTS patients had different clinical manifestation, mortality, and dialysis dependence patterns during hospitalization and even after hospital discharge, and thus should have different multidisciplinary teams to facilitate the implementation of enhanced recovery pathways. ${ }^{27}$ Because the incidence of AKI-D after major surgeries has increased in recent years, ${ }^{28}$ these findings provide a future direction of study for solving the unmet need of improving the mortality and outcomes in patients undergoing major surgeries with AKI-D. Furthermore, these results have significant implications for considering prevention strategies regarding different pathophysiology in the perioperative care of the patient undergoing major surgeries who has AKI-D.

In a study using data from the Ontario, Canada, universal health care databases to examine consecutive patients who had major elective surgery between 1995 and 2009, the incidence of AKI-D increased from $0.2 \%$ in 1995 to $0.6 \%$ in 2009 while $42 \%$ died within 90 days of surgery with no change in 90-day survival overtime and no change in chronic dialysis overtime among the patients surviving beyond 90 days. ${ }^{29}$ Lenihan et al examined AKI-D trends in a nationwide inpatient sample of individuals 75 years or 


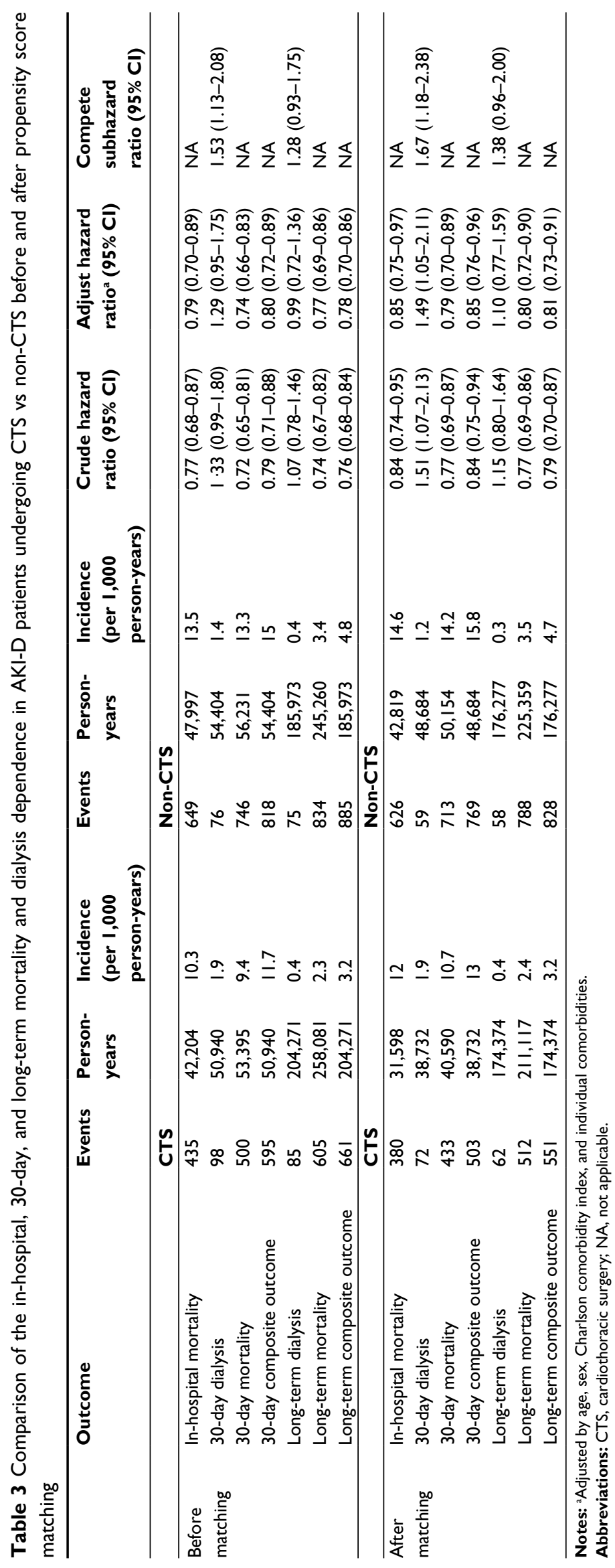



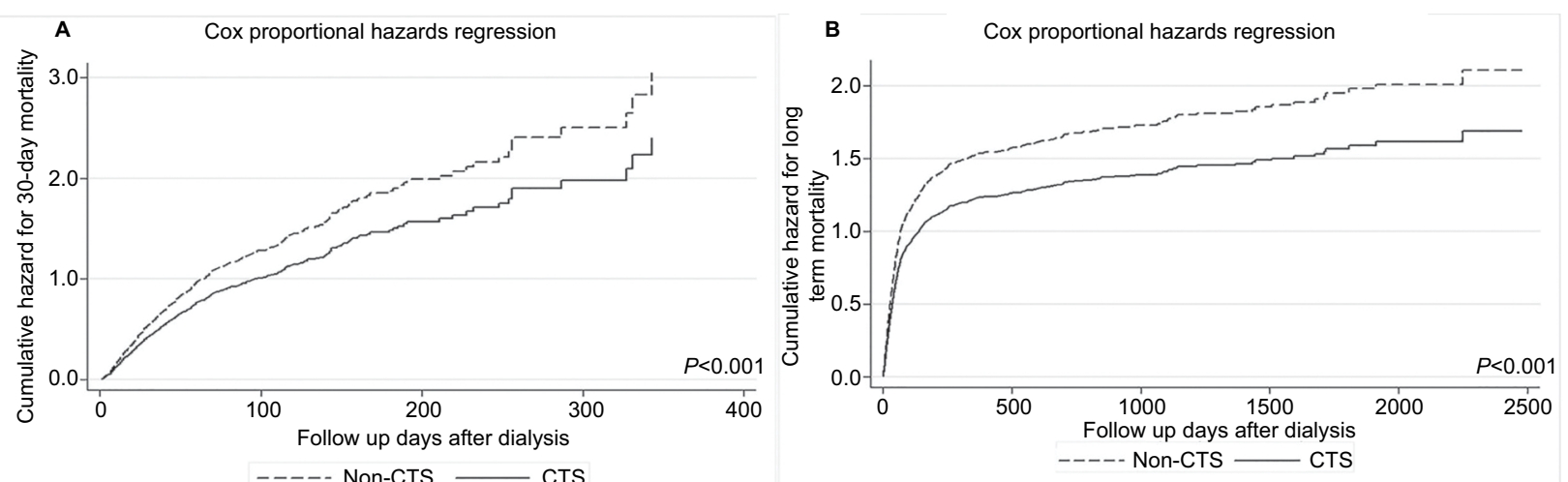

C

D
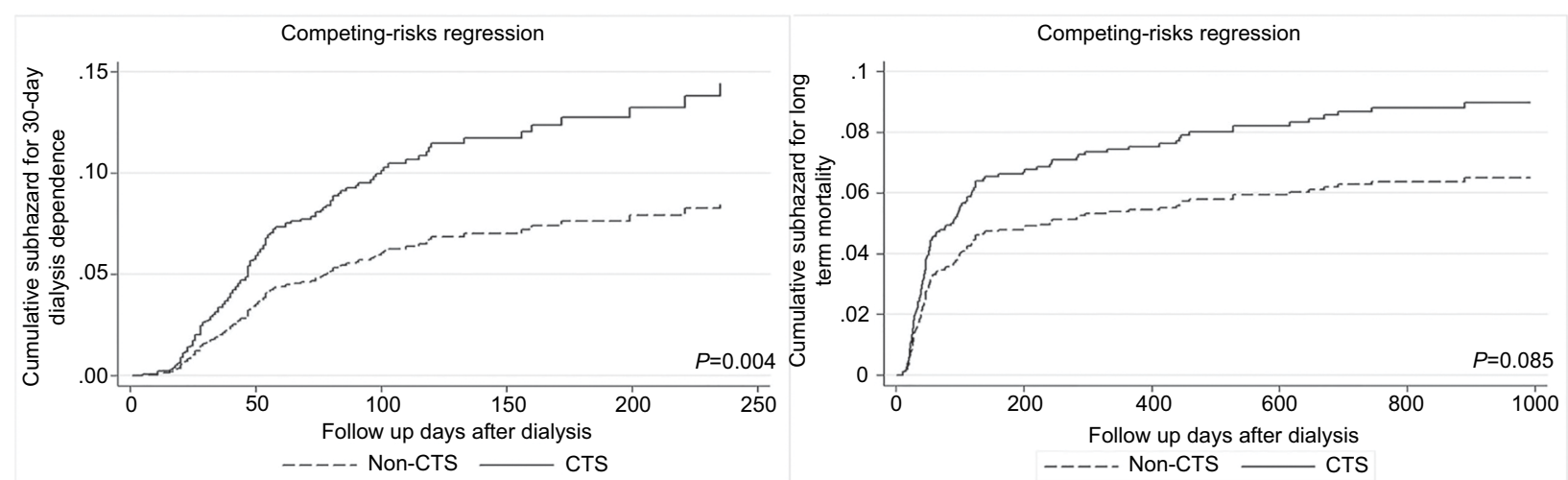

Figure 2 The mortality and competing risks regression of cumulative subhazard for dialysis dependence among different surgeries in 8,320 propensity score-matched surgical patients.

Notes: The hazard of short- and long-term mortality in different surgeries was shown in $(\mathbf{A})$ and $(\mathbf{B})$. The subhazard of short- and long-term dialysis dependence was shown in (C) and (D), while taking mortality as a competing risk.

Abbreviation: CTS, cardiothoracic surgery.

older admitted for cardiac surgery. They found that the risk of AKI-D was significantly higher in 2008 than in 1999 (OR, 2.23; 95\% CI 1.78-2.80), and the attributable risks for in-hospital mortality associated with AKI-D increased from $5 \%$ in 1999 to $14 \%$ in $2008 .{ }^{30}$ Compared with these studies, we studied longer-term outcomes than in-hospital or 90-day mortality and found an increased chance of postsurgical AKI-D together with increased long-term mortality overtime..$^{29,30}$ The growing incidence of AKI-D may be due to a heightened awareness and better detection of AKI with the introduction of the RIFLE (risk, injury, failure, loss, endstage renal disease) criteria in $2003^{31}$ and the AKIN (acute kidney injury network) criteria ${ }^{32}$ for AKI or the postsurgical cooperation of new RRT technology such as continuous RRT. However, even with adjustment for the higher burden of comorbid conditions and complications, the hazard ratio for mortality increased over the years, and this may be due to the shift toward more complex and higher risk procedures on sicker patients. ${ }^{33}$
The novel finding of our study is that post-CTS patients with AKI-D have lower in-hospital, 30-day, and long-term mortality than non-CTS patients. This finding fills important gaps in previous research. Because AKI-D is a perilous postsurgical complication associated with increased risk of mortality, dialysis dependence, resource utilization, and cost, ${ }^{8,34}$ action is urgently required to increase awareness of and to prevent AKI in patients undergoing different major surgeries and to facilitate renal recovery as early as possible to decrease mortality. Our study extends this finding by providing an overview of all surgical patients including CTS and non-CTS patients with the most severe form of AKI, AKI-D. CTS increases the likelihood of ischemia-reperfusion injury that temporarily compromises renal perfusion and causes renal dysfunction, ${ }^{35}$ the so-called cardiorenal syndrome type 1 in which patients are more likely to be weaned from dialysis..$^{36}$ Additionally, our study confirms recent findings that a substantial percentage of AKI-D survivors stay dialysis-dependent after the acute phase of their 
Table 4 The comparison between CTS and non-CTS patients after propensity score matching in NEP-AKI-D study ${ }^{23}$ database

\begin{tabular}{|c|c|c|c|c|c|c|}
\hline & \multirow{2}{*}{$\begin{array}{l}\text { CTS } \\
n=79\end{array}$} & \multirow{2}{*}{$\begin{array}{l}\text { Non-CTS } \\
n=154\end{array}$} & \multirow[t]{2}{*}{ SMD } & \multicolumn{3}{|c|}{ Propensity score-matched } \\
\hline & & & & $\begin{array}{l}\text { CTS } \\
n=72\end{array}$ & $\begin{array}{l}\text { Non-CTS } \\
\mathrm{n}=72\end{array}$ & SMD \\
\hline \multicolumn{7}{|l|}{ Comorbidities (during index } \\
\hline \multicolumn{7}{|l|}{ hospitalization) } \\
\hline Sepsis & $40(50.63 \%)$ & $129(83.77 \%)$ & 0.750 & $36(50.00 \%)$ & $62(86.11 \%)$ & 0.834 \\
\hline Azotemia & $26(32.91 \%)$ & 78 (50.65\%) & 0.364 & $22(30.56 \%)$ & $35(48.61 \%)$ & 0.402 \\
\hline Fluid overload & $54(68.35 \%)$ & II 3 (73.38\%) & 0.110 & $48(66.67 \%)$ & $52(72.22 \%)$ & 0.089 \\
\hline Electrolyte imbalance & $25(31.65 \%)$ & 47 (30.52\%) & 0.024 & $22(30.56 \%)$ & $21(29.17 \%)$ & 0.030 \\
\hline Acid-base imbalance & $26(32.91 \%)$ & 74 (48.05\%) & 0.311 & $25(34.72 \%)$ & $31(43.06 \%)$ & 0.170 \\
\hline Uremia & $3(3.80 \%)$ & $6(3.90 \%)$ & 0.005 & $3(4.17 \%)$ & $2(2.78 \%)$ & 0.075 \\
\hline Oliguria & $55(69.62 \%)$ & 109 (70.78\%) & 0.002 & $52(72.22 \%)$ & $57(79.17 \%)$ & 0.128 \\
\hline \multicolumn{7}{|l|}{ Laboratory data } \\
\hline \multicolumn{7}{|l|}{ At initiation of RRT } \\
\hline Glasgow coma scale & $8.63 \pm 4.22$ & $8.31 \pm 3.90$ & 0.079 & $8.65 \pm 4.36$ & $8.32 \pm 3.78$ & 0.108 \\
\hline $\mathrm{PaO}_{2} / \mathrm{FiO}_{2}$ & $272.49 \pm 180.28$ & $295.34 \pm 190.09$ & 0.123 & $281.89 \pm 184.93$ & $296.17 \pm 196.90$ & 0.122 \\
\hline Mean arterial pressure & $80.59 \pm 17.96$ & $82.16 \pm 21.17$ & 0.080 & $80.38 \pm 18.64$ & $81.20 \pm 22.18$ & 0.065 \\
\hline Serum PH & $7.37 \pm 0.11$ & $7.34 \pm 0.12$ & 0.261 & $7.37 \pm 0.11$ & $7.36 \pm 0.12$ & 0.089 \\
\hline Serum $\mathrm{HCO}_{3}$ & $20.60 \pm 5.06$ & $17.74 \pm 6.18$ & 0.507 & $20.4 I \pm 5.16$ & $18.20 \pm 5.20$ & 0.403 \\
\hline Albumin & $2.95 \pm 0.65$ & $2.58 \pm 0.66$ & 1.123 & $2.96 \pm 0.67$ & $2.64 \pm 0.72$ & 1.382 \\
\hline Total bilirubin & $2.19 \pm 3.70$ & $3.38 \pm 6.65$ & 0.220 & $2.25 \pm 3.86$ & $3.37 \pm 5.83$ & 0.229 \\
\hline Aspartate aminotransferase & $457.30 \pm 1,227.30$ & $593.83 \pm 1,625.58$ & 2.535 & $473.88 \pm 1,277.13$ & $429.89 \pm 1,009.50$ & 2.626 \\
\hline $\mathrm{Na}$ & $142.57 \pm 7.53$ & $|39.72 \pm 8.4|$ & 0.357 & $142.48 \pm 7.65$ & $139.04 \pm 8.16$ & 0.405 \\
\hline $\mathrm{K}$ & $4.42 \pm 0.97$ & $4.62 \pm 1.08$ & 0.193 & $4.45 \pm 0.95$ & $4.62 \pm 1.01$ & 0.131 \\
\hline White blood cell count & $|2,777.22 \pm 6,3| \mid .08$ & $14,052.93 \pm 8,926.50$ & 0.165 & $12,990.97 \pm 6,397.34$ & $|4,055.69 \pm 8,2| 9.88$ & 0.137 \\
\hline Blood urine nitrogen & $62.50 \pm 43.55$ & $79.56 \pm 45.49$ & 0.383 & $61.17 \pm 44.46$ & $78.17 \pm 41.37$ & 0.408 \\
\hline Creatinine & $3.53 \pm 2.04$ & $5.04 \pm 2.94$ & 0.597 & $3.44 \pm 1.77$ & $4.92 \pm 2.28$ & 0.700 \\
\hline Urine output & $547.44 \pm 671.81$ & $504.50 \pm 872.12$ & 0.055 & $536.08 \pm 683.60$ & $4 I 7.8 I \pm 56 I .45$ & 0.115 \\
\hline Lactic acid & $5.35 \pm 4.95$ & $5.90 \pm 6.31$ & 1.749 & $4.64 \pm 4.68$ & $5.78 \pm 7.13$ & 1.703 \\
\hline RRT modalities & & & 0.260 & & & 0.334 \\
\hline $\mathrm{CVVH}$ & 42 (85.7।\%) & $48(31.17 \%)$ & & 37 (5I.39\%) & I8 (25.00\%) & \\
\hline SLEDD/SLEDD-f & $4(5.06 \%)$ & 7 (4.55\%) & & $4(5.56 \%)$ & $5(6.94 \%)$ & \\
\hline IHD & $26(32.91 \%)$ & 84 (54.55\%) & & $24(33.33 \%)$ & 40 (55.56\%) & \\
\hline Mix methods & $7(8.86 \%)$ & 15 (9.74\%) & & $7(9.72 \%)$ & $9(12.50 \%)$ & \\
\hline Hospital stay length & $42.20 \pm 35.57$ & $44.13 \pm 34.78$ & 0.055 & $4 I .5 I \pm 36.15$ & $45.58 \pm 36.98$ & 0.127 \\
\hline Short-term mortality & $42(53.16 \%)$ & 93 (60.39\%) & 0.146 & 37 (5।.39\%) & $43(59.72 \%)$ & 0.139 \\
\hline Long-term mortality & $46(58.23 \%)$ & $104(67.53 \%)$ & 0.193 & $4 \mathrm{I}(56.94 \%)$ & $48(66.67 \%)$ & 0.170 \\
\hline $\begin{array}{l}\text { Long-term survival with renal } \\
\text { function recovery }\end{array}$ & 26 (32.9l\%) & $38(24.68 \%)$ & 0.182 & $25(34.72 \%)$ & $16(22.22 \%)$ & 0.245 \\
\hline
\end{tabular}

Note: Data presented as $\mathrm{n}(\%)$ or mean \pm SD.

Abbreviations: CTS, cardiothoracic surgery; CVVH, continuous venovenous hemofiltration; IHD, intermittent hemodialysis; NEP-AKI-D, nationwide epidemiology and prognosis of dialysis-requiring acute kidney injury; non-CTS, noncardiothoracic surgery; RRT, renal replacement therapy; SLEDD, sustained low-efficiency daily dialysis; SLEDD-f, sustained low-efficiency daily diafiltration; SMD, standardized mean difference.

illness has resolved. ${ }^{37,38}$ Even with lower mortality in CTS patients, this finding serves as an impetus to develop greatly needed measures that protect and restore kidney function as soon as possible after AKI-D.

This study provides updated evidence of the epidemiology of AKI in surgical patients together with mortality and dialysis dependence data, which is the missing puzzle piece for the quality control of surgical efficacy and performance in public health. Moreover, the results support the renal angina concept that AKI can be stratified into different surgery type categories ${ }^{39,40}$ Efforts of hemodynamic manipulations and close attention to intravenous resuscitation strategies including goal-directed therapy cannot be neglected to reduce AKI following major surgeries and its influence on postsurgical complications. ${ }^{41}$

These results provide direction for more personalized evaluation and optimized follow-up, taking into account surgery-specific characteristics. These findings also reinforce the importance of perioperative risk stratification for kidney injury and the implementation of strategies available to help 
prevent postsurgical AKI and long-term dialysis dependence. Furthermore, the findings indicate the potential for therapeutic interventions to attenuate the high burden of death and dialysis dependence after postsurgical AKI has occurred.

\section{Strengths and limitations}

A major strength of this study is the use of a large nationally representative database with exceptionally long renal followup; this gives us the ability to generalize and extrapolate the data with greater confidence. The NHIRD data are trustworthy because the database undergoes periodic detailed medical chart reviews and medical charge audits by professionals. ${ }^{42}$ The main limitation of our study is the lack of laboratory results for the national administrative dataset, leading to the possibility of residual confounding. Stricter control for estimated glomerular filtration rate or chronic kidney disease stage is crucial to derive more meaningful information. Besides, the CAKS database only included patients from ICU and that might bias our finding. Nevertheless, we validated the results in a different database with detailed laboratory data, and the results were similar in proving different manifestations in CTS and non-CTS patients in AKI. Furthermore, because this study was a retrospective observational analysis, a causal inference could not be derived. We did not assess the effect of the hospital- and surgeon-related factors such as hospital volume, location, emergent or elective surgery, prescribing physician, and level of experience. For the avoidance of the clustering effect, we used PSM together with multivariate adjustments and evaluated the model discrimination; we also attempted to increase the internal validity by using competing risk models. ${ }^{43,44}$ Because a high percentage of patients die after surgical AKI, we used competing risk analysis to examine the potential long-term dialysis dependence that would avoid confounding due to the "Will Rogers effect" of stage migration. ${ }^{45}$ Ours is also the first study to provide valuable long-term information related to kidney recovery following postsurgical AKI, given that follow-up for renal function recovery is almost nonexistent in recent clinical practice. Finally, while other investigators focused on the mortality issues in the specified surgical field having AKI, our results may provide data useful for generalized policy and practice frameworks for the proper perioperative care and future medical resource allocation. ${ }^{46}$

\section{Conclusions}

As the public health and economic burdens of surgery-related complications continue to grow, recognizing the entity of outpatient target-organ dysfunction is critical. Outpatient dialysis dependency is a pivotal clinical entity of postsurgical complications. In this nationwide population cohort of 106,573 surgical patients with AKI-D, we demonstrated an independent association between AKI-D and in-hospital, 30-day, and long-term mortality risk. Furthermore, CTS patients who have a high incidence of AKI-D have a lower risk of in-hospital, 30-day, and long-term mortality. Nonetheless, patients with CTS-related AKI-D had a higher risk of 30-day dialysis dependency, although their potential for long-term dialysis dependence was similar to that of patients with non-CTS AKI-D. In an era of increasing focus on patient-centered care, as well as continued reimbursement and quality improvement pressure, increased consideration for the management of renal function recovery toward an optimal team-based coordinated care after postsurgical AKI as an outpatient is warranted. The appropriate transition of patients undergoing surgery to follow-up in the outpatient setting with an emphasis on the renal function recovery and mitigation of mortality risk is justifiable in enhancing the care of the high-risk patients undergoing major surgeries. Physicians and policymakers should be aware of these results to access possible benefits of earlier initiation of recovery pathways and identify how to effectively reduce mortality and dialysis dependence for better perioperative management and thereby improve quality and reduce costs.

\section{Acknowledgments}

We would like to express our profound respect and sincere appreciation with deep sense of gratitude to Professor Likwang Chen, Institute of Population Health Sciences, National Health Research Institutes, Zhunan, Taiwan, for her kindness of supplying the data. We also would like to thank Professor Fu-Chang $\mathrm{Hu}$, Graduate Institute of Clinical Medicine and School of Nursing, National Taiwan University College of Medicine, Taipei, Taiwan, for his professional help of detailed statistical analysis. The study is partly based on data provided by Bureau of National Health Insurance, Department of Health, Taiwan. The interpretation and conclusions shown in this paper do not represent those of Bureau of National Health Insurance, Department of Health, National Health Research Institutes, or National Taiwan University Hospital. This study was supported by the Taiwan National Science Council grants 103-2314-B-002-026, 104-2314-B002-130, 104-2314-B-002-125-MY3, 105-2314-B-002-045 and National Taiwan University Hospital 106-S3466, 106FTN20, 106-P02, UN106-014, 106-S3582, 105-M3195, 105P05, VN105-04, 105-S3061, VN104-07, 104-S2718. This study was also supported by Ta-Tung Kidney Foundation, 
and the Mrs Hsiu- Chin Lee Kidney Research Foundation, Taipei, Taiwan. We thank the staff of the Eighth Core Lab, Department of Medical Research, National Taiwan University Hospital for technical support during the study. We also appreciate and thank all the members of the Taiwan Clinical Trial Consortium, TCTC grant MOST 106-2321-B-182-002, 105-2314-B-002-045, which are listed in alphabetical order of their affiliation names: Cardinal Tien Hospital: Kuo-Cheng Lu; Chi-Mei Medical Center, Liouying: Jian-Jhong Wang; Chi-Mei Medical Center, Yongkang: Wei-Chih Kan; China Medical University Hospital: Chiu-Ching Huang, Che-Yi Chou, Ya-Fei Yang; Dalin Tzu-Chi Hospital: Jen-Pi Tsai; Far Eastern Memorial Hospital: Hung-Yuan Chen; InternationalHarvard Statistical Consulting Company: Fu-Chang $\mathrm{Hu}$; Kaohsiung Chang Gung Memorial Hospital: Chien-Te Lee, Jin-Bor Chen, Chih-Hsiung Lee, Wen-Chin Lee, Lung-Chih Li, Te-Chuan Chen; Kaohsiung Municipal Ta-Tung Hospital: Hugo You-Hsien Lin; Keelung Chang Gung Memorial Hospital: Chin-Chan Lee, Chiao-Yin Sun, MD, Heng-Chih Pan; Linkou Chang Gung Memorial Hospital: Yung-Chang Chen, Ming-Yang Chang, Chang-Chyi Jenq, Chan-Yu Lin, ChihHsiang Chang, Tsung-Yu Tsai; Lin-Shin Hospital: ChengMin Chen; Lotung Poh-Ai Hospital: En-Tzu Lin; Luodong Saint Mary's Hospital: Chih-Chung Shiao; Mackay Memorial Hospital: Chih-Jen Wu, Cheng-Jua Lin; Mackay Memorial Hospital Taitung Branch: Feng-Chi Kuo; Mennonite Christian Hospital: I-Chieh Tsai; Min-Sheng General Hospital: ChihJen Weng; National Health Research Institutes: LiKwang Chen; National Taiwan University Hospital: Kwan-Dun Wu, Tzong-Shinn Chu, Shuei-Liong Lin, MD, PhD, Vin-Cent Wu, Chun-Fu Lai; National Taiwan University Hospital Bei-Hu Branch: Tai-Shuan Lai; National Taiwan University Hospital Hsin Chu Branch: Wei-Shun Yang; National Taiwan University Hospital Yun-Lin Branch: Yung-Ming Chen, Tao-Min Huang; New Taipei City Hospital Sanchong Branch: WenDing Hsu; Shin-Kong Wo Ho-Su Memorial Hospital: JyhGang Leu, Jui-Ting Chang; Sin-Ren Hospital: Hung-Hsiang Liou; Taichung Veteran General Hospital: Kuo-Hsiung Hsu, Ming-Ju Wu, Chun-Te Huang; Taichung Veteran General Hospital Chiayi Branch: Zi-hong You; Taipei City Hospital Heping Branch: Chao-Fu Chang; Taipei Medical University Hospital: Tzen-Wen Chen, Hsi-Hsien Chen, Fan-Chi Chang, Yen-Chung Lin, Mai-Szu Wu, Chih-Chin Kao; Taipei Tzu Chi Hospital: Szu-Chun Hung, Ko-Lin Kuo, Che-Hsiung Wu; Taipei Veterans General Hospital: Der-Cherng Tarng, Kuo-Hua Lee; Taoyuan General Hospital, Ministry of Health and Welfare: Wei-Jie Wang, Jui-Hsiang Lin. We especially thank Professor Ji-Tseng Fang, Chang Gung University Col- lege of Medicine, Taoyuan, Taiwan; Kidney Research Center, Department of Nephrology, Chang Gung Memorial Hospital, Taiwan, for his extraordinary work and leadership skills in establishing CAKS cohort.

\section{Disclosure}

The authors report no conflicts of interest in this work.

\section{References}

1. Hoste EA, Clermont G, Kersten A, et al. RIFLE criteria for acute kidney injury are associated with hospital mortality in critically ill patients: a cohort analysis. Crit Care. 2006;10(3):R73.

2. Mandelbaum T, Scott DJ, Lee J, et al. Outcome of critically ill patients with acute kidney injury using the Acute Kidney Injury Network criteria. Crit Care Med. 2011;39(12):2659-2664.

3. Bellomo R. Acute renal failure. Semin Respir Crit Care Med. 2011;32(5): 639-650.

4. Thakar CV. Perioperative acute kidney injury. Adv Chronic Kidney Dis. 2013;20(1):67-75.

5. Kim CS, Oak CY, Kim HY, et al. Incidence, predictive factors, and clinical outcomes of acute kidney injury after gastric surgery for gastric cancer. PLoS One. 2013;8(12):e82289.

6. Lee EH, Kim HR, Baek SH, et al. Risk factors of postoperative acute kidney injury in patients undergoing esophageal cancer surgery. J Cardiothorac Vasc Anesth. 2014;28(4):936-942.

7. Chertow GM, Burdick E, Honour M, Bonventre JV, Bates DW. Acute kidney injury, mortality, length of stay, and costs in hospitalized patients. J Am Soc Nephrol. 2005;16(11):3365-3370.

8. Hobson C, Ozrazgat-Baslanti T, Kuxhausen A, et al. Cost and mortality associated with postoperative acute kidney injury. Ann Surg. 2015;261(6):1207-1214.

9. Nisula S, Kaukonen KM, Vaara ST, et al. Incidence, risk factors and 90-day mortality of patients with acute kidney injury in Finnish intensive care units: the FINNAKI study. Intensive Care Med. 2013;39(3):420-428.

10. Srisawat N, Lawsin L, Uchino S, Bellomo R, Kellum JA, BEST Kidney Investigators. Cost of acute renal replacement therapy in the intensive care unit: results from The Beginning and Ending Supportive Therapy for the Kidney (BEST Kidney) study. Crit Care. 2010;14(2):R46.

11. Parikh A, Shaw A. The economics of renal failure and kidney disease in critically ill patients. Crit Care Clin. 2012;28(1):99-111.

12. Lin YF, Ko WJ, Chu TS, et al. The 90-day mortality and the subsequent renal recovery in critically ill surgical patients requiring acute renal replacement therapy. Am J Surg. 2009;198(3):325-332.

13. Coca SG, Singanamala S, Parikh CR. Chronic kidney disease after acute kidney injury: a systematic review and meta-analysis. Kidney Int. 2012;81(5):442-448.

14. Doi K, Rabb H. Impact of acute kidney injury on distant organ function: recent findings and potential therapeutic targets. Kidney Int. 2016;89(3):555-564.

15. Bihorac A, Brennan M, Ozrazgat-Baslanti T, et al. National surgical quality improvement program underestimates the risk associated with mild and moderate postoperative acute kidney injury. Crit Care Med. 2013;41(11):2570-2583.

16. Ingraham AM, Richards KE, Hall BL, Ko CY, Cy K. Quality improvement in surgery: the American College of Surgeons National Surgical Quality Improvement Program approach. Adv Surg. 2010;44:251-267.

17. Cohen ME, Ko CY, Bilimoria KY, et al. Optimizing ACS NSQIP modeling for evaluation of surgical quality and risk: patient risk adjustment, procedure mix adjustment, shrinkage adjustment, and surgical focus. J Am Coll Surg. 2013;217346(2):336:-346.

18. Cohen ME, Liu Y, Ko CY, Hall BL. Improved surgical outcomes for ACS NSQIP hospitals over time: evaluation of hospital cohorts with up to 8 years of participation. Ann Surg. 2016;263(2):267-273. 
19. Ho Chan WS. Taiwan's healthcare report 2010. EPMA J. 2010;1(4): 563-585.

20. $\mathrm{Wu} \mathrm{VC}, \mathrm{Wu} \mathrm{CH}$, Huang TM, et al. Long-term risk of coronary events after AKI. J Am Soc Nephrol. 2014;25(3):595-605.

21. Wu CS, Lai MS, Gau SS, Wang SC, Tsai HJ. Concordance between patient self-reports and claims data on clinical diagnoses, medication use, and health system utilization in Taiwan. PLoS One. 2014;9(12):e112257.

22. Lindenauer PK, Pekow P, Wang K, Mamidi DK, Gutierrez B, Benjamin EM. Perioperative beta-blocker therapy and mortality after major noncardiac surgery. $N$ Engl J Med. 2005;353(4):349-361.

23. Shiao CC, Wu PC, Wu VC, et al. Nationwide epidemiology and prognosis of dialysis-requiring acute kidney injury (NEP-AKI-D) study: design and methods. Nephrology. 2016;21(9):758-764.

24. Grams ME, Sang Y, Coresh J, et al. Acute kidney injury after major surgery: a retrospective analysis of veterans health administration data. Am J Kidney Dis. 2016;67(6):872-880.

25. Mishra PK, Luckraz H, Nandi J, et al. Long-term quality of life postacute kidney injury in cardiac surgery patients. Ann Card Anaesth. 2018;21(1):41-45.

26. Fine JP, Gray RJ. A proportional hazards model for the subdistribution of a competing risk. J Am Stat Assoc. 1999;94(446):496-509.

27. Stone AB, Yuan CT, Rosen MA, et al. Barriers to and facilitators of implementing enhanced recovery pathways using an implementation framework: a systematic review. JAMA Surg. 2018;153(3):270.

28. Siew ED, Davenport A. The growth of acute kidney injury: a rising tide or just closer attention to detail? Kidney Int. 2015;87(1):46-61.

29. Siddiqui NF, Coca SG, Devereaux PJ, et al. Secular trends in acute dialysis after elective major surgery - 1995 to 2009. CMAJ. 2012;184(11):1237-1245.

30. Lenihan CR, Montez-Rath ME, Mora Mangano CT, Chertow GM, Winkelmayer WC. Trends in acute kidney injury, associated use of dialysis, and mortality after cardiac surgery, 1999 to 2008. Ann Thorac Surg. 2013;95(1):20-28.

31. Bellomo R, Ronco C, Kellum JA, Mehta RL, Palevsky P, Acute Dialysis Quality Initiative Workgroup. Acute renal failure - definition, outcome measures, animal models, fluid therapy and information technology needs: the Second International Consensus Conference of the Acute Dialysis Quality Initiative (ADQI) Group. Crit Care. 2004;8(4):R204-212.

32. Mehta RL, Kellum JA, Shah SV, et al. Acute Kidney Injury Network: report of an initiative to improve outcomes in acute kidney injury. Crit Care. 2007;11(2):R31.
33. Kolhe NV, Muirhead AW, Wilkes SR, Fluck RJ, Taal MW. National trends in acute kidney injury requiring dialysis in England between 1998 and 2013. Kidney Int. 2015;88(5):1161-1169.

34. Bihorac A. Acute kidney injury in the surgical patient: recognition and attribution. Nephron. 2015;131(2):118-122.

35. Ishani A, Nelson D, Clothier B, et al. The magnitude of acute serum creatinine increase after cardiac surgery and the risk of chronic kidney disease, progression of kidney disease, and death. Arch Intern Med. 2011;171(3):226-233.

36. Bagshaw SM, Cruz DN, Aspromonte N, et al. Epidemiology of cardiorenal syndromes: workgroup statements from the 7th ADQI Consensus Conference. Nephrol Dial Transplant. 2010;25(5):1406-1416.

37. VA/NIH Acute Renal Failure Trial Network, Palevsky PM, Zhang JH, et al. Intensity of renal support in critically ill patients with acute kidney injury. N Engl J Med. 2008;359(1):7-20.

38. Wald R, Mcarthur E, Adhikari NK, et al. Changing incidence and outcomes following dialysis-requiring acute kidney injury among critically ill adults: a population-based cohort study. Am J Kidney Dis. 2015;65(6):870-877.

39. Chertow GM, Lazarus JM, Christiansen CL, et al. Preoperative renal risk stratification. Circulation. 1997;95(4):878-884.

40. Chawla LS, Goldstein SL, Kellum JA, Ronco C. Renal angina: concept and development of pretest probability assessment in acute kidney injury. Crit Care. 2015;19:93.

41. O'Neal JB, Shaw AD, Billings FT, Ftt B. Acute kidney injury following cardiac surgery: current understanding and future directions. Crit Care. 2016;20(1): 187

42. Chen YC, Su YC, Lee CC, Huang YS, Hwang SJ. Chronic kidney disease itself is a causal risk factor for stroke beyond traditional cardiovascular risk factors: a nationwide cohort study in Taiwan. PLoS One. 2012;7(4):e36332.

43. Dignam JJ, Zhang Q, Kocherginsky M. The use and interpretation of competing risks regression models. Clin Cancer Res. 2012;18(8):2301-2308.

44. Wolbers M, Blanche P, Koller MT, Witteman JC, Gerds TA. Concordance for prognostic models with competing risks. Biostatistics. 2014;15(3):526-539.

45. Feinstein AR, Sosin DM, Wells CK. The Will Rogers phenomenon. Stage migration and new diagnostic techniques as a source of misleading statistics for survival in cancer. $N$ Engl J Med. 1985;312(25):1604-1608.

46. Fair allocation of intensive care unit resources. American Thoracic Society. Am J Respir Crit Care Med. 1997;156(4 Pt 1):1282-1301. 


\section{Supplementary materials}

Table SI Factors identified as predictors of AKI-D during index admission for the propensity score matching ${ }^{\mathrm{a}}$

\begin{tabular}{|c|c|c|}
\hline Predictive variables & Odds ratio $(95 \% \mathrm{Cl})$ & $P$-value \\
\hline Advanced CKD & $32.740(20.191-53.509)$ & $<0.00 I^{b}$ \\
\hline Cardiac arrest & $5.115(1.966-12.221)$ & $<0.00 I^{b}$ \\
\hline Use of ECMO & $4.993(4.236-5.890)$ & $<0.00 \mathrm{I}^{\mathrm{b}}$ \\
\hline Acute respiratory distress syndrome & $4.860(4.240-5.567)$ & $<0.00 I^{b}$ \\
\hline Hypovolemic shock & $3.472(2.944-4.083)$ & $<0.00 I^{b}$ \\
\hline Hospital stay length & $3.320(2.96 \mathrm{I}-3.722)$ & $<0.00 \mathrm{I}^{\mathrm{b}}$ \\
\hline Repeat intubation & $1.733(1.123-2.622)$ & $0.011^{b}$ \\
\hline Chest tube insertion & 1.691 (1.443-1.976) & $<0.00 I^{b}$ \\
\hline Severe sepsis & $1.624(1.34 I-1.963)$ & $<0.00 \mathrm{I}^{\mathrm{b}}$ \\
\hline Rheumatologic disease & I.62I (1.023-2.466) & $0.03 \mathrm{I}^{\mathrm{b}}$ \\
\hline Hemopericardium & $1.449(0.973-2.099)$ & 0.058 \\
\hline Prolonged mechanical ventilation & $1.397(1.254-1.556)$ & $<0.00 I^{b}$ \\
\hline Gl bleeding & $1.382(1.120-1.694)$ & $0.002^{\mathrm{b}}$ \\
\hline Charlson comorbidity index score & $1.307(1.235-1.383)$ & $<0.00 I^{b}$ \\
\hline Diabetes & $1.279(I . I I 2-|.47|)$ & $0.00 \mathrm{I}^{\mathrm{b}}$ \\
\hline Surgical type (CVS vs others) & $1.200(1.034-1.389)$ & $0.016^{\mathrm{b}}$ \\
\hline Sex & $1.101(0.989-1.226)$ & 0.079 \\
\hline Age & $1.009(1.005-1.012)$ & $<0.00 I^{b}$ \\
\hline Pneumonia & $0.832(0.692-0.998)$ & $0.049^{b}$ \\
\hline Pleural effusion & $0.734(0.560-0.950)$ & $0.022^{\mathrm{b}}$ \\
\hline Myocardial infarction & $0.693(0.539-0.884)$ & $0.004^{b}$ \\
\hline Atrial fibrillation & $0.687(0.530-0.879)$ & $0.004^{b}$ \\
\hline Cerebrovascular disease & $0.623(0.502-0.767)$ & $<0.00 \mathrm{I}^{\mathrm{b}}$ \\
\hline Chronic obstructive pulmonary disease & $0.563(0.474-0.666)$ & $<0.00 I^{b}$ \\
\hline Dementia & $0.557(0.373-0.806)$ & $0.003^{b}$ \\
\hline Hemiplegia or paraplegia & $0.330(0.135-0.698)$ & $0.007^{b}$ \\
\hline Malignancy & $0.328(0.236-0.452)$ & $<0.00 \mathrm{I}^{\mathrm{b}}$ \\
\hline
\end{tabular}

Notes: a Goodness of fit test: variance inflation faction of all factors (all $<2$ ); adjust generalized $R^{2}=0.295$; area under the receiver operating characteristic curve $=0.906$ (0.899-0.913); this model does not pass the modified Hosmer-Lemeshow test. ${ }^{b} P<0.05$.

Abbreviations: AKI-D, acute kidney injury requiring dialysis; CKD, chronic kidney disease; CVS, cardiovascular surgery; ECMO, extra-corporeal membrane oxygenation; $\mathrm{Gl}$, gastrointestinal.

Table S2 Incidence of AKI-D and its related crude mortality in 106,573 surgical patients stratified by four major surgeries

\begin{tabular}{|c|c|c|c|c|c|c|c|c|c|c|}
\hline Patients & Surgery type & 2001 & 2002 & 2003 & 2004 & 2005 & 2006 & 2007 & Total & $P$ for trend \\
\hline Patient & All & 14,424 & 14,886 & 13,615 & 15,328 & 15,775 & 16,212 & 16,333 & 106,573 & \\
\hline \multirow[t]{4}{*}{ numbers } & Cardiothoracic & 2,037 & 2,120 & 1,937 & 2,969 & 3,738 & 3,753 & 3,781 & 20,335 & \\
\hline & Esophagus & 2,964 & 3,123 & 2,909 & 2,663 & 2,315 & 2,194 & 2,100 & 18,268 & \\
\hline & Intestine & 3,644 & 3,962 & 3,700 & 3,920 & 4,082 & 4,142 & 4,207 & 27,657 & \\
\hline & Liver & 5,779 & 5,681 & 5,069 & 5,776 & 5,640 & 6,123 & 6,245 & 40,313 & \\
\hline AKI-D, & All & $167(1.16)$ & $196(1.32)$ & $194(1.43)$ & $249(1.62)$ & $344(2.18)$ & $376(2.32)$ & $335(2.05)$ & $\mathrm{I}, 86 \mathrm{I}(\mathrm{I} .75)$ & \\
\hline \multirow[t]{4}{*}{ n (\%) } & Cardiothoracic & $30(1.47)$ & $60(2.83)$ & $50(2.58)$ & $120(4.04)$ & $188(5.03)$ & $212(5.65)$ & $199(5.26)$ & $859(4.22)$ & $<0.001$ \\
\hline & Esophagus & $53(1.79)$ & $43(1.38)$ & $57(1.96)$ & 37 (1.39) & $55(2.38)$ & $58(2.64)$ & $44(2.10)$ & $347(1.90)$ & 0.008 \\
\hline & Intestine & 54 (I.48) & $61(1.54)$ & $56(1.5 \mathrm{I})$ & $54(1.38)$ & $53(1.30)$ & $73(1.76)$ & $53(1.26)$ & $404(1.46)$ & 0.661 \\
\hline & Liver & $30(0.52)$ & $32(0.56)$ & $3 I(0.6 I)$ & $38(0.66)$ & $48(0.85)$ & $33(0.54)$ & $39(0.62)$ & $25 I(0.62)$ & 0.378 \\
\hline Mortality & All & I $33(79.64)$ & I 32 (67.35) & 134 (69.07) & 161 (64.66) & $224(65.12)$ & 244 (64.89) & $218(65.07)$ & $1246(66.95)$ & \\
\hline in AKI-D, & Cardiothoracic & $22(73.33)$ & $30(50.00)$ & $30(60.00)$ & $70(58.33)$ & $\mathrm{II} 0(58.5 \mathrm{I})$ & $122(57.55)$ & $116(58.29)$ & $500(58.21)$ & 0.789 \\
\hline \multirow[t]{3}{*}{ n (\%) } & Esophagus & $46(86.79)$ & $33(76.74)$ & $46(80.70)$ & 27 (72.97) & $46(83.64)$ & $49(84.48)$ & 37 (84.09) & $284(81.84)$ & 0.771 \\
\hline & Intestine & $43(79.63)$ & $44(72.13)$ & $38(67.86)$ & 37 (68.52) & 37 (69.8I) & $49(67.12)$ & 39 (73.58) & $287(71.04)$ & 0.385 \\
\hline & Liver & $22(73.33)$ & $25(78.13)$ & $20(64.52)$ & 27 (7I.05) & 31 (64.58) & $24(72.73)$ & $26(66.67)$ & I 75 (79.72) & 0.435 \\
\hline
\end{tabular}

\footnotetext{
Abbreviation: AKI-D, acute kidney injury requiring dialysis.
} 
Table S3 The risk factors of short- and long-term mortality in surgical patients

\begin{tabular}{|c|c|c|c|c|}
\hline \multirow[t]{2}{*}{ Risk factors } & \multicolumn{2}{|l|}{ Short-term mortality } & \multicolumn{2}{|l|}{ Long-term mortality } \\
\hline & Parameter estimate & Hazard ratio $(95 \% \mathrm{Cl})$ & Parameter estimate & Hazard ratio $(95 \% \mathrm{Cl})$ \\
\hline$\overline{A K I-D}$ & 1.7638 & $5.83(5.38-6.32)$ & 1.2845 & $3.6 I(3.37-3.87)$ \\
\hline Acute respiratory distress syndrome & 1.2702 & $3.56(3.17-4.00)$ & 1.0597 & $2.89(2.64-3.16)$ \\
\hline Repeat intubation & 1.1378 & $3.12(2.48-3.92)$ & 0.6363 & $\mathrm{I} .89(1.54-2.3 \mathrm{I})$ \\
\hline Hypovolemic shock & 0.932 & $2.54(2.27-2.84)$ & 0.7595 & $2.14(1.95-2.34)$ \\
\hline Chest tube insertion & 0.4487 & $1.57(1.40-1.75)$ & 0.2963 & $1.34(1.23-1.47)$ \\
\hline Stroke & 0.3789 & $1.46(1.16-1.83)$ & 0.3096 & $1.36(1.15-1.62)$ \\
\hline Hemopericardium & 0.3428 & $1.41(1.08-1.84)$ & - & \\
\hline Gl bleeding & 0.1861 & $1.20(1.06-1.37)$ & - & \\
\hline Severe sepsis & 0.1387 & $1.15(1.04-1.26)$ & - & \\
\hline Rheumatologic disease & - & & 0.2498 & $1.28(1.00-1.64)$ \\
\hline Charlson comorbidity index score & 0.1399 & $1.15(1.13-1.18)$ & 0.1365 & $1.15(1.12-1.18)$ \\
\hline Malignancy & - & & 0.1681 & $1.18(1.02-1.37)$ \\
\hline Liver disease & - & & 0.1105 & $1.12(1.00-1.24)$ \\
\hline Sex & 0.091 & $1.10(1.01-1.19)$ & 0.105 & I.II (I.04-I.I8) \\
\hline Age & 0.0142 & $1.01(1.01-1.02)$ & 0.0205 & $1.02(1.02-1.02)$ \\
\hline
\end{tabular}

Abbreviations: -, not applicable; AKI-D, acute kidney injury requiring dialysis; $\mathrm{Gl}$, gastrointestinal.

Table S4 CTS and non-CTS patients matched

\begin{tabular}{|c|c|c|c|c|c|c|}
\hline \multirow[t]{2}{*}{ The matched parameters } & \multirow{2}{*}{$\begin{array}{l}\text { CTS } \\
n=79\end{array}$} & \multirow{2}{*}{$\begin{array}{l}\text { Non-CTS } \\
n=I 54\end{array}$} & \multirow[t]{2}{*}{ SMD } & \multicolumn{3}{|c|}{ Propensity score-matched } \\
\hline & & & & $\begin{array}{l}\text { CTS } \\
n=72\end{array}$ & $\begin{array}{l}\text { Non-CTS } \\
n=72\end{array}$ & SMD \\
\hline Male sex & $5 \mathrm{I}(64.56 \%)$ & 109 (70.78\%) & 0.063 & $48(66.67 \%)$ & 47 (65.28\%) & 0.029 \\
\hline Age & $66.36 \pm 14.59$ & $65.4 I \pm I 5.75$ & 0.133 & $65.50 \pm 14.59$ & $66.89 \pm \mid 5.21$ & 0.093 \\
\hline Charlson comorbidity index score & $6.20 \pm 3.04$ & $6.31 \pm 3.20$ & 0.033 & $6.14 \pm 3.10$ & $6.24 \pm 3.22$ & 0.031 \\
\hline APACHE II score & $21.81 \pm 7.61$ & $23.01 \pm 6.31$ & 0.171 & $21.86 \pm 7.89$ & $22.14 \pm 6.38$ & 0.039 \\
\hline SOFA score & $12.53 \pm 3.96$ & $12.31 \pm 3.92$ & 0.056 & $12.29 \pm 3.87$ & $12.25 \pm 3.55$ & 0.011 \\
\hline
\end{tabular}

Note: Data presented as $\mathrm{n}(\%)$ or mean $\pm \mathrm{SD}$.

Abbreviations: APACHE II, acute physiology and chronic health evaluation II; CTS, cardiothoracic surgery; SMD, standardized mean difference; SOFA, sequential organ failure assessment. 
A

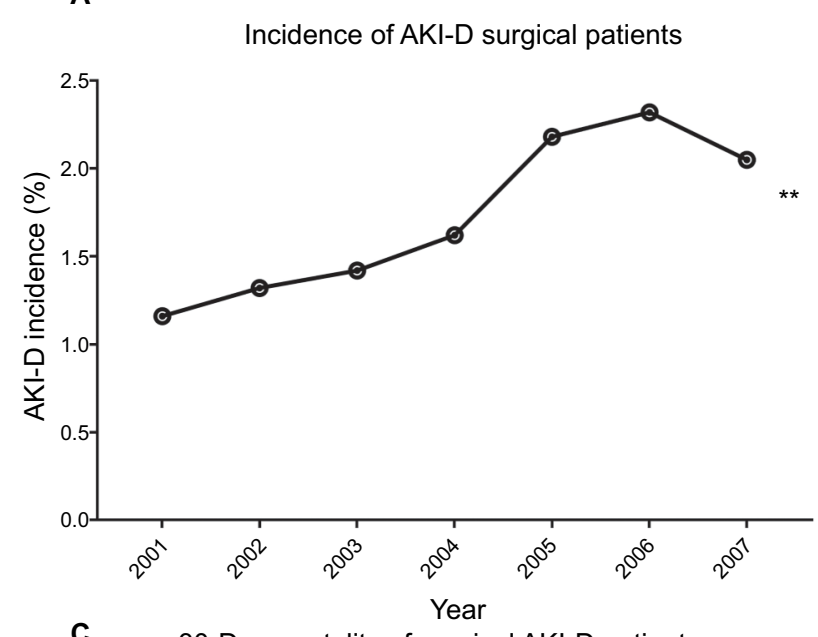

C 30-Day mortality of surgical AKI-D patients

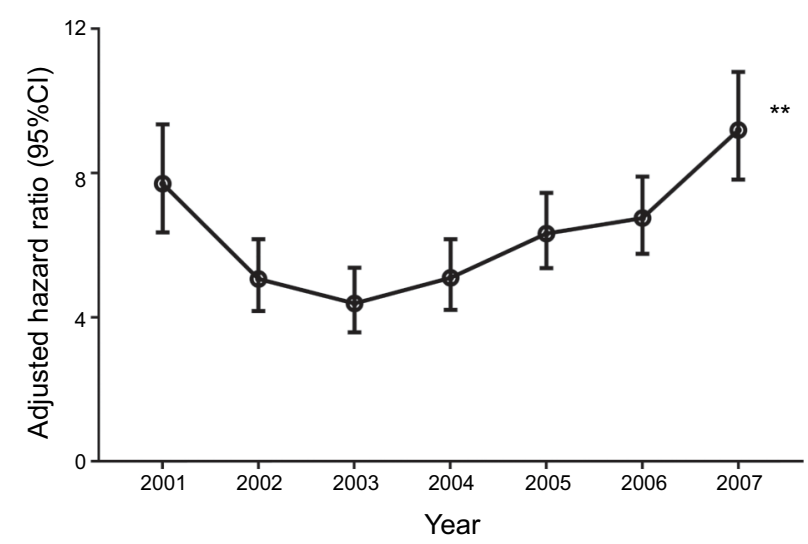

E

Long-term mortality of surgical AKI-D patients

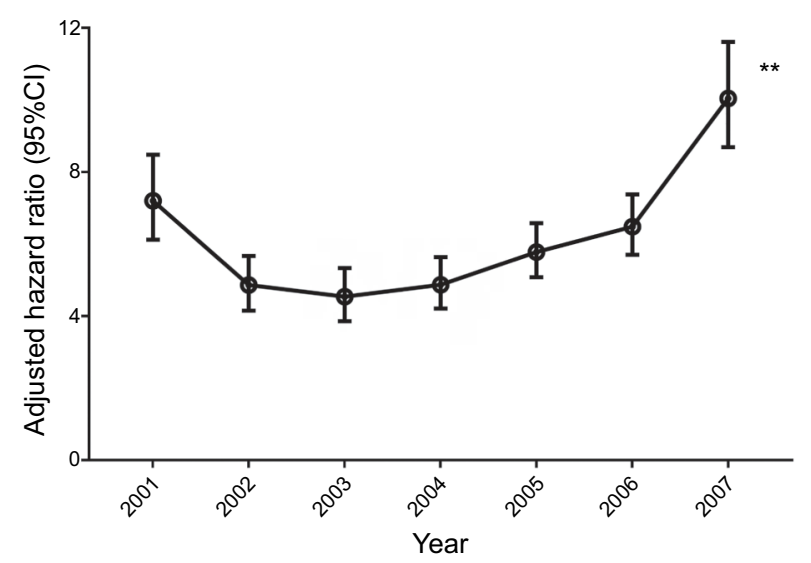

B

Incidence of AKI-D surgical patients

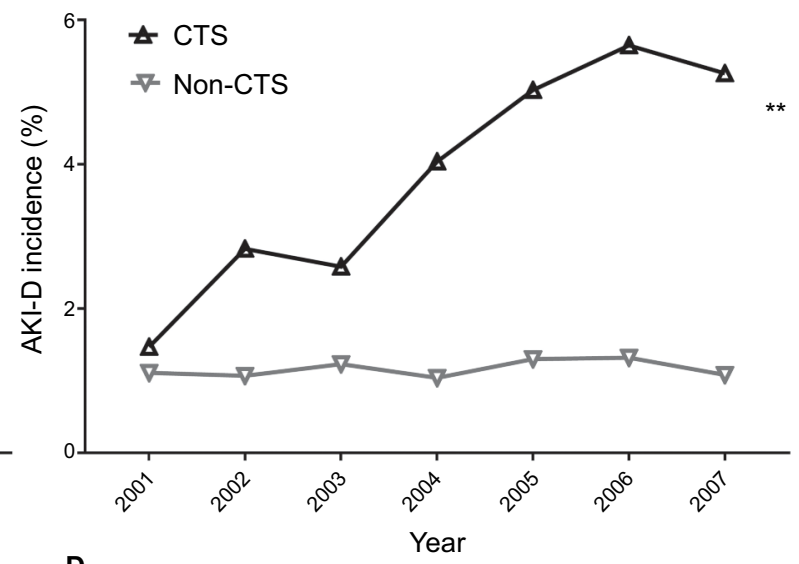

30-Day mortality of surgical AKI-D patients

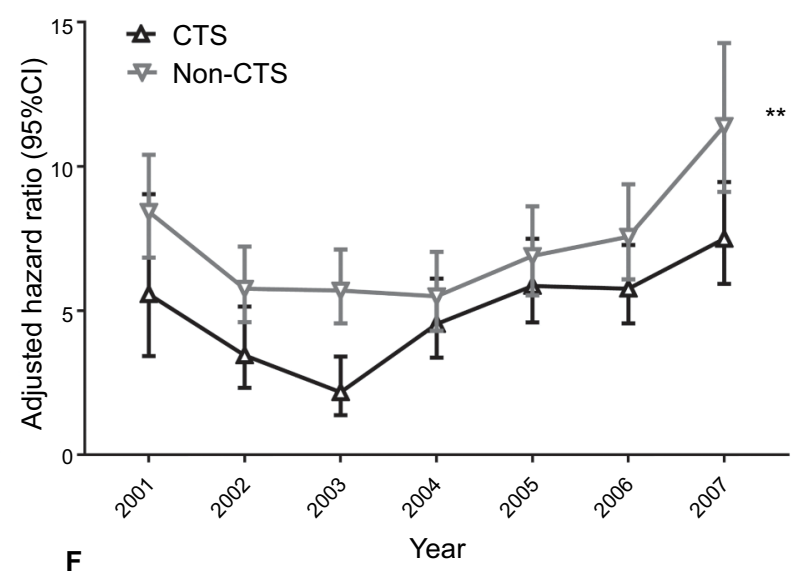

Long-term mortality of surgical AKI-D patients

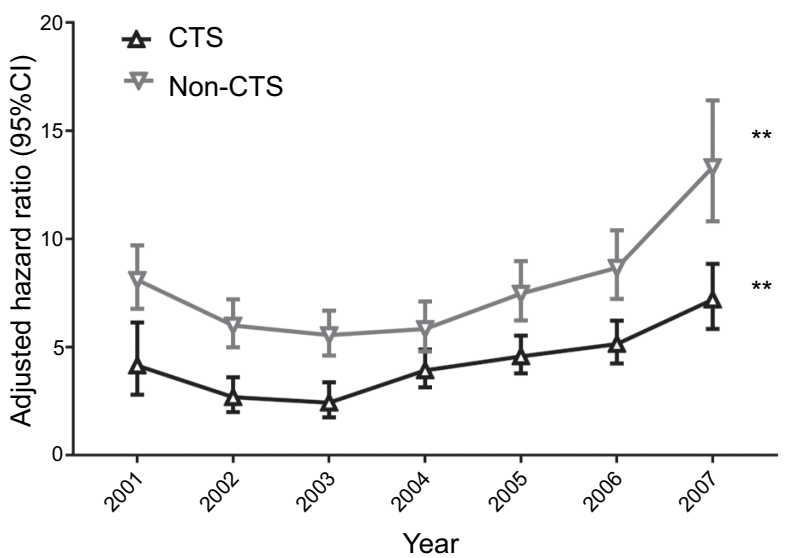

Figure SI The trend of yearly AKI-D incidence in 106,573 surgical patients and an annually adjusted hazard ratio of 30-day and long-term mortality in I,86I surgical AKI-D patients, stratified by the surgical type.

Notes: The annual incidence of AKI-D was shown in (A) and was compared in different surgical types in (B). The adjusted hazard ratio of 30-day mortality of surgical AKI-D patients was shown in (C) and was compared in different surgical types in (D), while the adjusted hazard ratio of long-term mortality of surgical AKI-D patients was shown

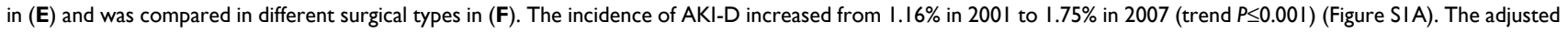
hazard ratio for 30-day mortality increased from 7.7 ( $95 \% \mathrm{Cl} 6.35-9.35)$ in $200 \mathrm{I}$ to 9.19 (95\% Cl 7.82-10.9) in 2007 (Figure SIC), and long-term mortality increased from 7.2 ( $95 \% \mathrm{Cl} 6.12-8.48)$ in $200 \mathrm{I}$ to 10.04 ( $95 \% \mathrm{Cl} 8.69-\mathrm{II} .6 \mathrm{I})$ in 2007 (Figure SIE). Post-CTS patients had a higher incidence of AKI-D compared with non-CTS patients (Figure SIB). However, the 30-day and long-term mortality were higher in non-CTS patients (Figure SID and F). $* * P<0.001$.

Abbreviations: AKI-D, acute kidney injury requiring dialysis; CTS, cardiothoracic surgery. 


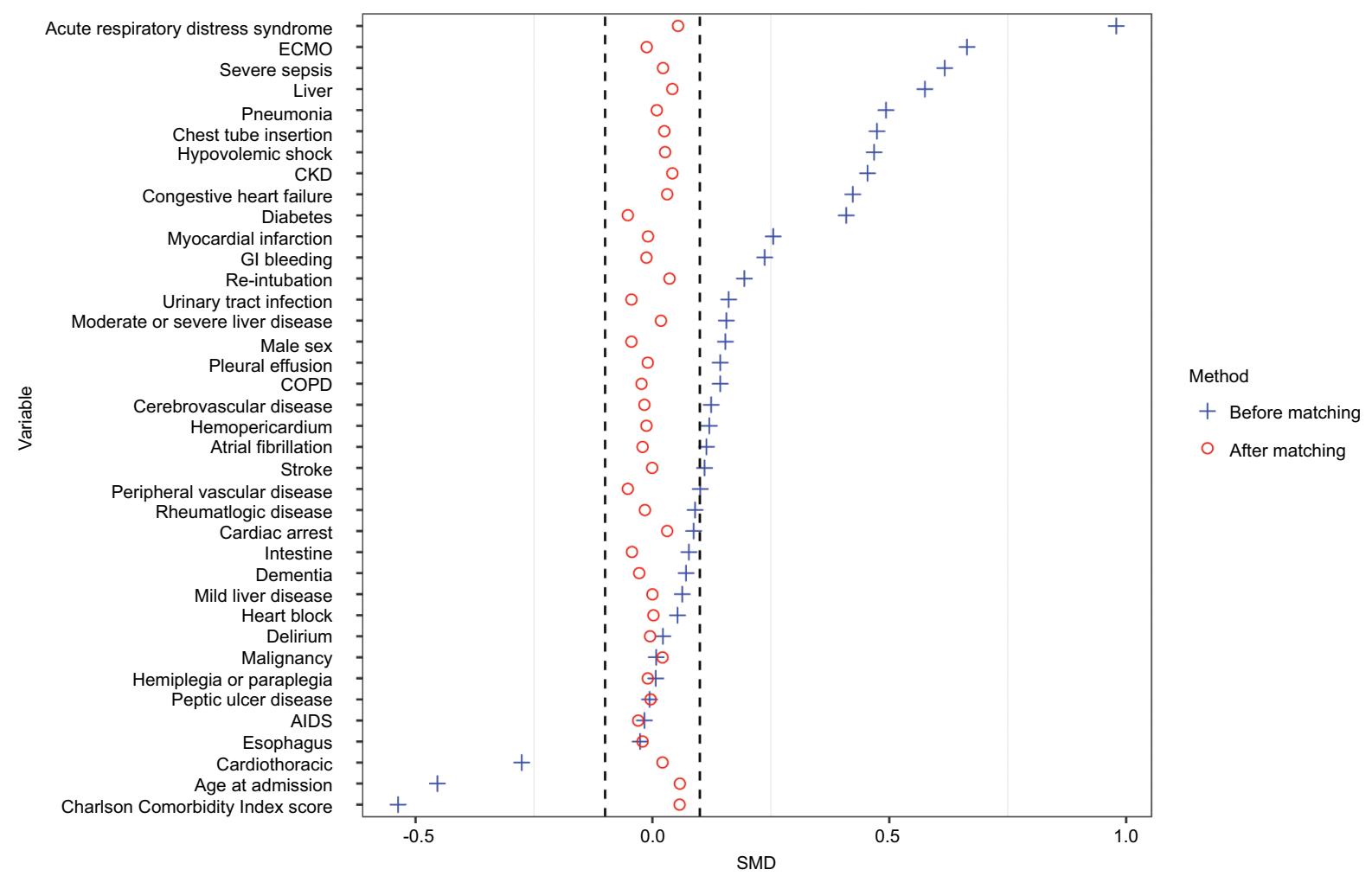

Figure S2 The standardized mean difference (SMD) of the variables before and after the propensity score matching. After the matching, the SMD of all variables was $<0.1$. Abbreviations: CKD, chronic kidney disease; COPD, chronic obstructive pulmonary disease; ECMO, extracorporeal membrane oxygenation; GI, gastrointestinal.

\section{Publish your work in this journal}

Clinical Epidemiology is an international, peer-reviewed, open access, online journal focusing on disease and drug epidemiology, identification of risk factors and screening procedures to develop optimal preventative initiatives and programs. Specific topics include: diagnosis, prognosis, treatment, screening, prevention, risk factor modification,

Submit your manuscript here: https://www.dovepress.com/clinical-epidemiology-journa systematic reviews, risk and safety of medical interventions, epidemiology and biostatistical methods, and evaluation of guidelines, translational medicine, health policies and economic evaluations. The manuscript management system is completely online and includes a very quick and fair peer-review system, which is all easy to use. 